\title{
Gloss and Goniophotometric Curves of Woven Fabrics
}

\author{
By Koji Nihira*, Tsuneyo Tsuboi*, and Toshihiro Gunji**, Members, TMSJ \\ *Faculty of Engineering, Meiji University, Kanagawa-ken. \\ **Nitto Boseki Co., Ltd., Tokyo
}

Based on the Journal of the Textile Machinery Society of Japan, Transactions, Vol. 30, No. 4, T77-84 (1977)

\begin{abstract}
To calculate the specularly reflected light distribution curve of fabrics a simplified model of the parallel arrangement of elliptical columus was used, and the calculated curves were compared with measurements.

The following results are obtained:

(1) The same tendency is found for the calculated specular reflection as for the measurement of fabrics.
\end{abstract}

(2) A method is proposed to evaluate the quality and quantity of the gloss of fabrics.

\section{Introduction}

The previous paper ${ }^{[1]}$ reported the results of a theoretical calculation to approximate the diffusely reflected light distribution curves by using a modified model having the surface on which elliptical cylinders are regularly arranged. Due to an insufficient assumption given to the diffusion, no results highly correlated to the experimental results were obtained, but relevant results were found qualitatively.

In this paper two cases are assumed: in one the fibers are parallel and in the other perpendicular to the incident luminous flux, regardless of warp or weft direction. As only the parallel component of the reflected light is measured, the fabric surface is simulated by a smooth surface in the parallel case, and by bundls of parallel fibers in the perpendicular case. Also, the specularly reflected light distribution curve is assumed to be able to get by superposing the results in there two cases. Both the cross section and the envelope of there cases are assumed to be elliptical.

\section{Calculation of the Specularly Reflected Light Distribu- tion Curve}

Since the specularly reflected light distribution curve consists of the specular reflection on the primary boundary surface, the minnute roughness of the surface must not be neglected.

So, in our study, two models are considered: the first model has such a surface as elliptical cylinders are regularly arrenged and the second has a smooth elliptical surface as assumed in the previous paper ${ }^{[1]}$.

\subsection{The model having many circular cylinders}

The specularly reflected distribution curve will be calculated when parallel incident luminous flux is cast perpendicularly to the axis of fiber bundles forming an elliptical cylinder. Each single fiber is assumed to be a circular cylinder having infinitesimally small diameters $r$, and arranged parallel to the axis of the elliptical cylinder as shown in Fig. 1. There is a plane surface between elliptical cylinders when they form the fabric surface. But the reflection from this plane is neglected because it gives no effect on the reflected light distribution curve.

The cross section of an elliptical cylinder is given by the following equation:

$$
x^{2}+\frac{y^{2}}{k^{2}}=1
$$

As the observing plane is perpendicular to the axis of an elliptical cylinder, the analytical calculation is made in twodimensions, and only with a elliptical cylinder. Also, the length of the major axis of the elliptical cylinder is taken as a unit. The number of circular cylinders covering the surface of an elliptical cylinder is assumed to be $N$. The peripheral length of the cross section of an elliptical cylinder from $x=$ 0 to 1 is calculated by

$$
l=\int_{0}^{1} \sqrt{\frac{1-\left(1-k^{2}\right) x^{2}}{1-x^{2}}} d x \fallingdotseq 2 N r
$$

Assuming that the incident angle of the parallel luminous flux is $\varphi$, the cylinder portion on which the specular reflection occurs is $\widehat{\mathrm{AB}}$, as shown in Fig. 2.

The length of $\widehat{\mathrm{AB}}$ located at $\mathrm{P}$, apart from the origin by $x$,

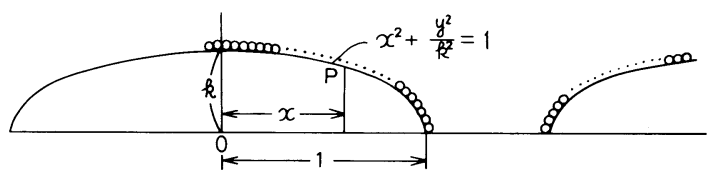

Fig. 1 Elliptical cylinders covered with circular cylinders 


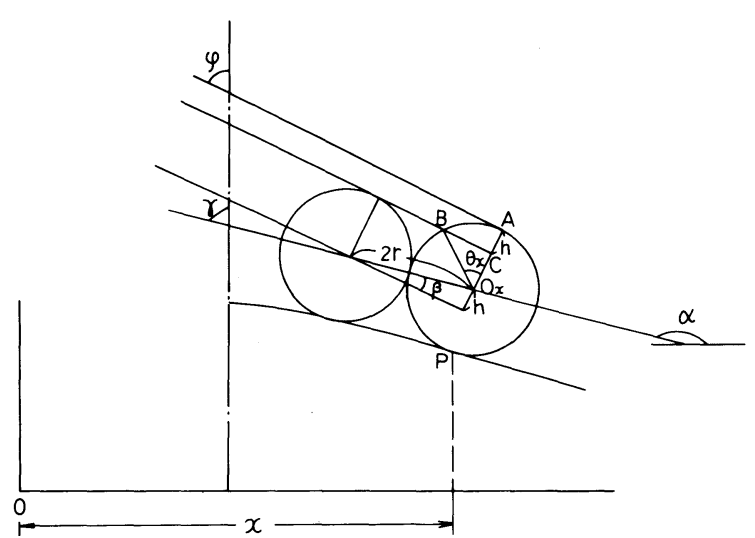

Fig. 2 Relation between circular cylinders

is calculated as

$$
\frac{d y}{d x}=\frac{-k x}{\sqrt{1-x^{2}}}
$$

Assuming that a straight line passing through two centers of neighbouring cylinders and $x$-axis forms the angle $\alpha$, that and $y$-axis the angle $\gamma$, and that and the incident ray the angle $\beta$, we have

$$
\begin{aligned}
& \tan \alpha=\frac{d y}{d x}=\frac{-k x}{\sqrt{1-x^{2}}} \\
& \alpha=\gamma+\frac{\pi}{2}=\tan ^{-1} \frac{-k x}{\sqrt{1-x^{2}}} \\
& \beta=\gamma-\varphi
\end{aligned}
$$

The projection $h$ of $\widehat{\mathrm{AB}}$ onto the radius $\mathrm{O}_{\mathrm{x}} \mathrm{A}$ is calcultated by

$$
h=2 r \cdot \sin \beta=2 r \cdot \sin (\gamma-\varphi)
$$

By eqs. (5) and (7) the central angle $\theta_{x}$ covered by $\overparen{\mathrm{AB}}$ is calculated as:

$$
\begin{aligned}
h= & \frac{2 r}{\sqrt{1-x^{2}+k^{2} x^{2}}}\left(-k x \sin \varphi+\sqrt{1-x^{2}} \cos \varphi\right) \\
\therefore & r-h=r\left(1-\frac{2}{\sqrt{1-x^{2}+k^{2} x^{2}}}(-k x \sin \varphi\right. \\
& \left.+\sqrt{1-x^{2}} \cos \varphi\right) \\
\therefore & \frac{r-h}{r}=1-\frac{2}{\sqrt{1-x^{2}+k^{2} x^{2}}}(-k x \sin \varphi \\
& \left.+\sqrt{1-x^{2}} \cos \varphi\right) \quad \cos \theta_{x}=\frac{r-h}{r} \\
\therefore & \theta_{x}=\cos ^{-1}\left\{1-\frac{2}{\sqrt{1-x^{2}+k^{2} x^{2}}}(-k x \sin \varphi\right. \\
& \left.\left.+\sqrt{1-x^{2}} \cos \varphi\right)\right\}
\end{aligned}
$$

Figure 3 illustrates this $\theta_{x}$ when $k$ is $0.1,0.4$ and 0.8 and $\varphi$ is $45^{\circ}, 60^{\circ}$. It is shown that the portion contributing to the specular reflection decreases with incresasing $k$ and $\varphi$.

The specular reflection of the circular cylinder at point $\mathbf{P}$ is shown in Fig. 4. The infinitesimally small surface $d s$ on

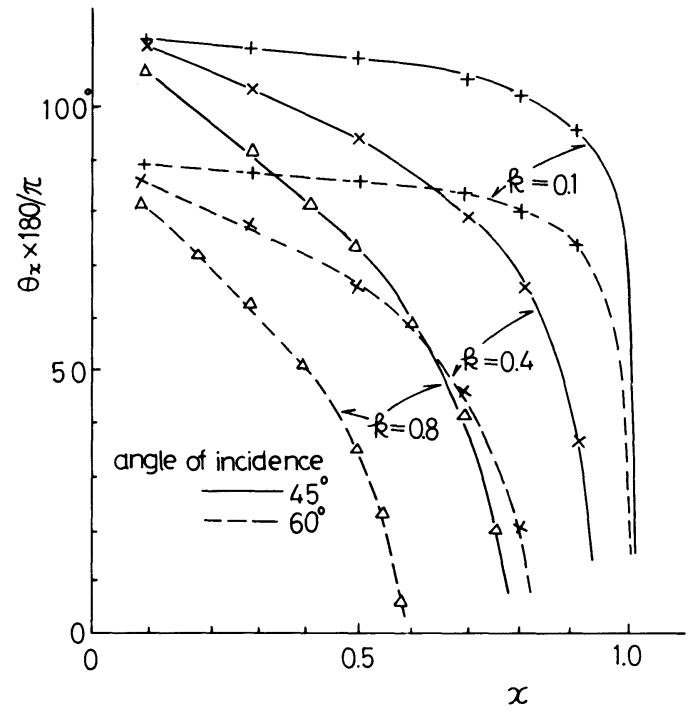

Fig. 3 Relation between $\theta_{x}$ and $x$

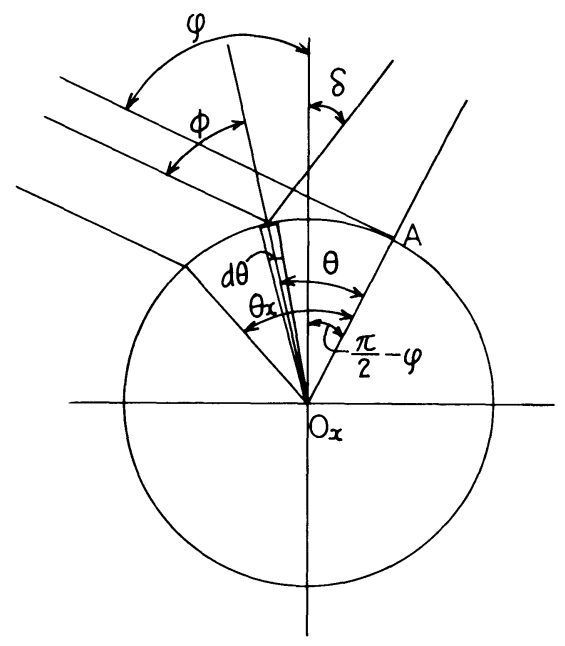

Fig. 4 Light on a circular cylinder

the surface is $d s=r \cdot d \theta$. The receiving angle $\delta$ on $d s$ is:

$$
\delta=2\left(\frac{\pi}{2}-\theta\right)-\varphi
$$

As the incident angle $\phi$ onto $d s$ is $(\pi / 2-\theta)$, the specular reflection $d I$ on $d s$, is expressed by:

$$
d I=f\left(\frac{\pi}{2}-\theta\right) \cdot \cos \left(\frac{\pi}{2}-\theta\right) \cdot r \cdot d \theta
$$

when the Fresnel coefficient is assumed to be $f(\pi / 2-\theta)$. If the refractive index $n$ is known, $f(\pi / 2-\theta) \cdot \cos (\pi / 2-\theta)$ is determined as shown in Fig. 5. $d I$ is only obtained within the angle of $\theta_{x}$.

The solid lines in Fig. 6 show the values of $\theta_{x}$ calculated for $\varphi=60^{\circ}$ and $k=0.1,0.4$ and 0.8 . As $k$ increases, the cir- 


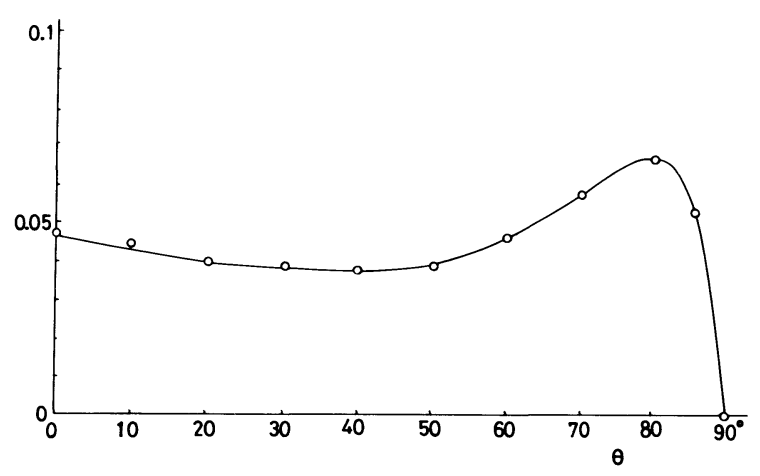

Fig. 5 The Curve of $f(\theta) \cdot \cos \theta$ $(n=1.53, f(\theta)$ is Fransnel's coefficient)

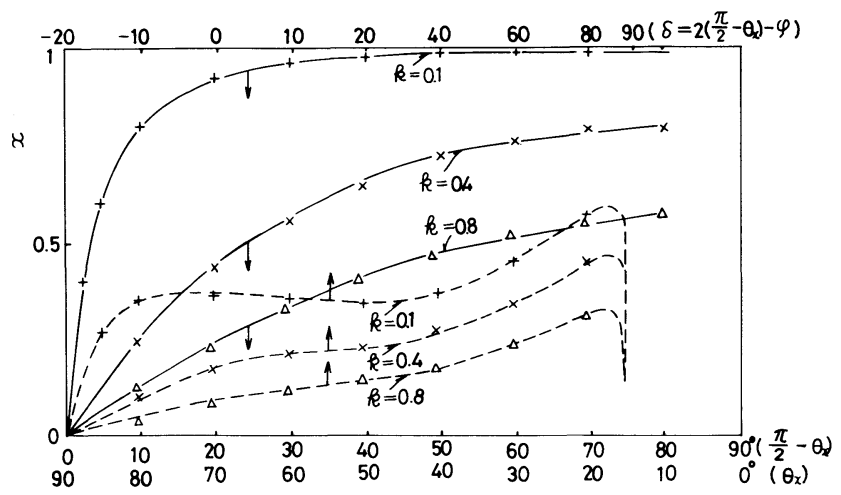

Fig. 6 When the elliptical cylindrical surface is covered with infinitesimally small circular cylinder

cular cylinders contributing to the specular reflection decrease their number. Assuming that the curvature of the elliptical cylinder is negligible, and that the number of circular cylinders on the elliptical cylinder is proportional to $x$, its value indicates the number of infinitesimally small surfaces inclined by $\theta_{x}$. The angle $\left(\pi / 2-\theta_{x}\right)$ incident onto the infinitesimally surface and the angle $\delta=2\left(\pi / 2-\theta_{x}\right)-\varphi$ receiving to the observer are also shown in Fig. 6. Dotted curves are obtained by multiplying $x$ corresponding to $\left(\pi / 2-\theta_{x}\right)$ by $f\left(\pi / 2-\theta_{x}\right) \cdot \cos \left(\pi / 2-\theta_{x}\right)$. These curves give the intensity of the reflected light under various receiving angles $\delta$. The similarity of these curves regardless of $k$ would be attributed to arranging the circular cylinders on the elliptical cylinder.

\subsection{The model having elliptical cylinders}

Consider an elliptical cylinder shown in Fig. 7. The surface roughness is changed by varing the value $k$. After taking the length of the major axis of the elliptical cylinder as a unit, the incident luminous flux is calculated in two-dimensions.

The infinitesimally small length $d l$ at point $\mathbf{P}$ apart from the origin by $x$ can be calculated as

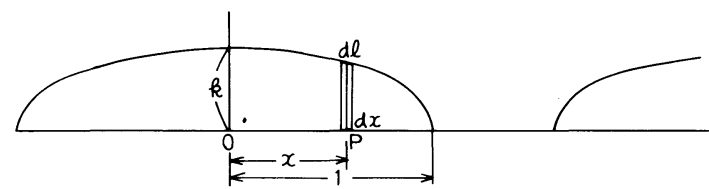

Fig. 7 Elliptical cylindrical model

$$
\frac{d l}{d x}=\sqrt{1+\frac{k^{2} x^{2}}{1-x^{2}}}
$$

Figure 8 indicates the curves of $d l / d x$ rapidly increasing with the increare of $k$ at large $x$. Since the major axis is taken as 1 , the area of the infinitesimally small surface $d s$ is $d l$.

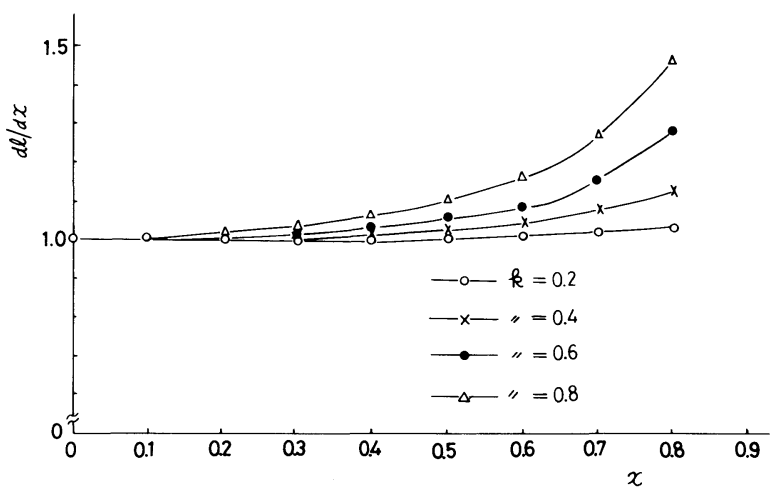

Fig. 8 The curves of $d l / d x$

Assuming that the angle $d s$ to the abscissa is $\gamma$, the receiving angle $\delta$ is Fig. 9.

$$
\delta=\varphi+2 \gamma, \quad \text { at } 0<x<1,
$$

or

$$
\delta=\varphi-2 \gamma, \quad \text { at } 0>x>-1
$$

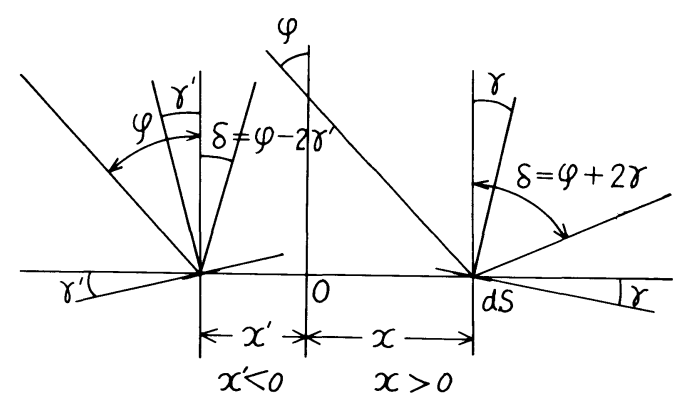

Fig. 9 The incident and the receiving angle on the infinitesimally small surface 


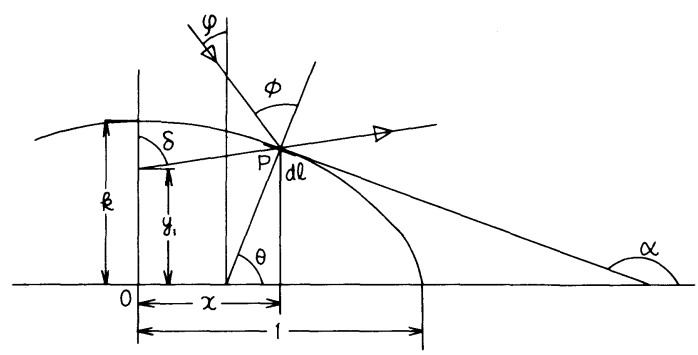

Fig. 10 The incident and the receiving angle on the infinitesimally small surface

The angle $\gamma$ is shown in Fig. 10 and is given by

$$
\gamma=\pi-\alpha, \alpha=\pi-\gamma,
$$

Thus, from eq. (3) we have

$$
\begin{aligned}
& \frac{d y}{d x}=\frac{-k x}{\sqrt{1-x^{2}}}=\tan \alpha=\tan (\pi-\gamma)=-\tan \gamma \\
& \gamma=\tan ^{-1} \frac{k x}{\sqrt{1-x^{2}}}
\end{aligned}
$$

As shown in Fig. 10, the infinitesimally small surface $d s=d l$ is not at the center 0 . But as its deviation $y$ is negligibly small, all the small surfaces are assumed to locate at the measuring center 0 .

Also, the intensity of reflected light of parallel luminous flux is assumed to be $\Delta F$ by neglecting the Fresnel coefficient $f(\phi)$ and observed coefficient $\cos \phi$.

The inclination of $n$-th small surface $\gamma_{n}$ and that for $(n+$ 1)th $\gamma_{n+1}$ are as shown in Fig. 11:

$$
\begin{aligned}
& \gamma_{n}=\tan ^{-1} \frac{k n}{\sqrt{1-n^{2}}} \\
& \gamma_{n+1}=\tan ^{-1} \frac{k(\mathrm{n}+1)}{\sqrt{1-(n+1)^{2}}}
\end{aligned}
$$

So,

$$
\begin{aligned}
\Delta \gamma & =\gamma_{n+1}-\gamma_{n} \\
& =\tan ^{-1} \frac{k(n+1)}{\sqrt{1-(n+1)^{2}}}-\tan ^{-1} \frac{k n}{\sqrt{1-n^{2}}}
\end{aligned}
$$

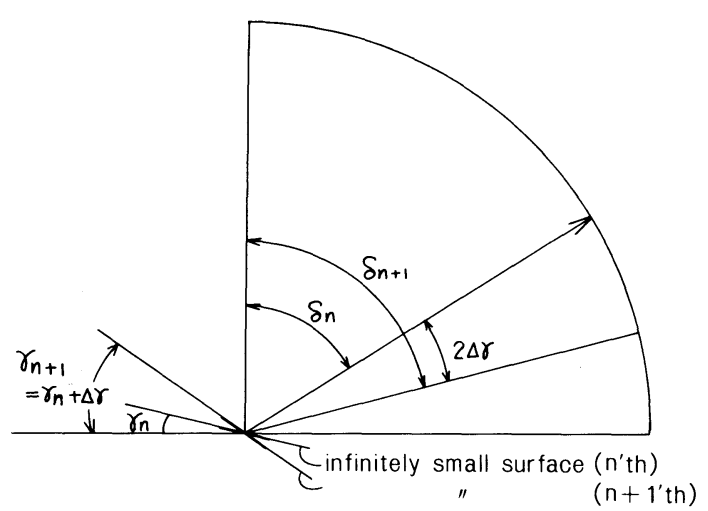

Fig. 11 The relation between $\Delta x$ and $\delta$ of the specular reflection

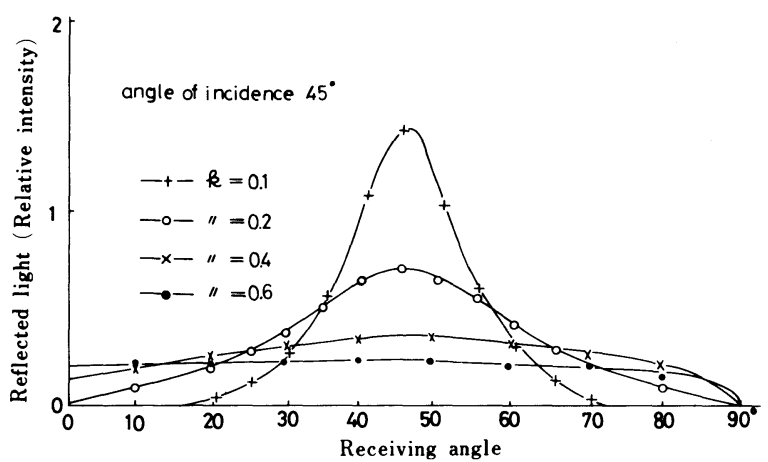

Fig. 12 Calculated specularly reflected light distribution curve (1)

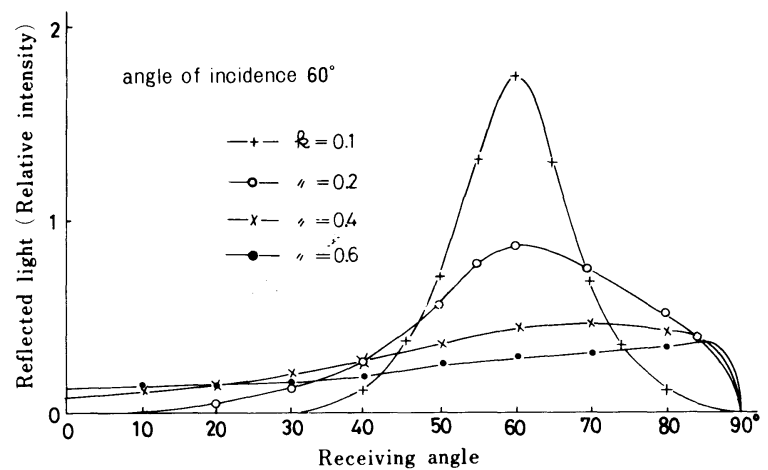

Fig. 13 Calculated specularly reflected light distribution curve (2)

Therefore, the difference between the receiving angles

$$
\delta_{n+1}-\delta_{n}=\varphi+2 \gamma_{n+1}-\left(\varphi+2 \gamma_{n}\right)=2 \Delta \gamma
$$

The luminous flux $\Delta F$ reflected from the $\mathrm{n}$-th infinitesimally surface is assumed uniformly extending through the angle $2 \Delta \gamma$, which can be considered to be the solid angle.

Therefore, the luminous intensity $I$ within the angle $2 \Delta \gamma$ is given by

$$
\Delta I=\frac{\Delta F}{2 \Delta \gamma}
$$

Since $\Delta F$ is constant, $\Delta I$ is proportional to $1 / 2 \Delta \gamma$, the distribution of which is determined by the receiving angle $\delta$.

The specularly reflected light distribution curve is finally determined by multiplying the corresponding value $\delta$ by the above-mentioned correction coefficient $d l / d x$.

Figures 12 and 13 show the examples of specularly reflected light distribution for the incident angle of $45^{\circ}$ and $60^{\circ}$, in which $x$ was varied in 0.05 steps and $k$ were $0.1,0.2,0.4$ and 0.6 and the refractive index was assumed to be 1.53 .

\section{Comaprison of Calculation with Measured Results}

Figures 14 and 15 illustrate the reflected light distribution 


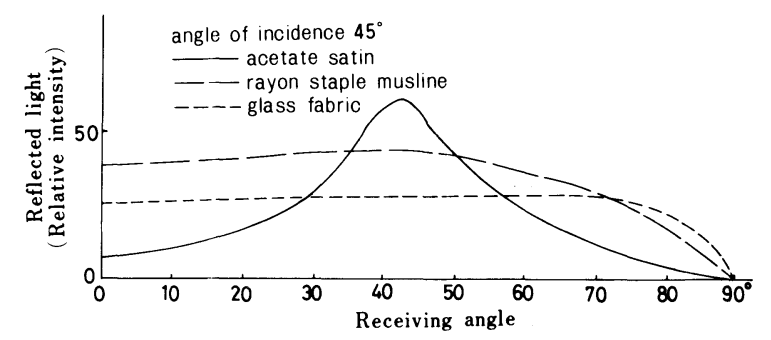

Fig. 14 Measured reflected light distribution curve (1)

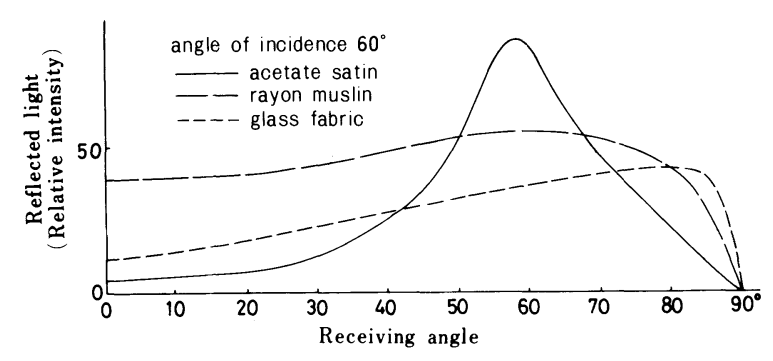

Fig. 15 Measured reflected light distribution curve (2)

curves of acetate satin, rayon muslin and glass fiber fabrics shown in Table 1 for $\varphi=45^{\circ}$ and $60^{\circ}$. These curves were determined by the sum total of specular and diffuse components. The curves of acetate satin are similar to the calculated for $k=0.1$ or 0.2 , those of rayon muslin fabric similar to the calculated for $k=0.4$, and those of glass fiber fabrics similar to the calculated for $k=0.6$.

It is natural that the measured curves are similar to those of calculation, because fabrics expected to follow the theoretical assumption are selected but other fabrics also showed similar tendency.

Summarizing these, two conclusions have been confirmed:

1) The fabric surface is considered optically as the arrangement of smooth elliptical cylinders, and its roughness can be expressed by the length of the minor axis of the elliptical cylinder $k$.

2) For small $k$, the maximum reflection appears at the receiving angle equal to the incident angle.

As $k$ becomes large, the receiving angle giving the maximum reflection moves toward $90^{\circ}$, and the specular component is distributed through the wide receiving angle.

The similar tendency can be recognized for materials other than textile fabrics such as paper.

\section{Reflected Light Distribution Curve and Luster}

Authors proposed some methods ${ }^{[3-5]}$ to evaluate quantitatively the luster.

Here, a proposal is also made on the basis of calculated results of the diffusely reflected light distribution curve. It shows that the diffusely reflected light follows the Lambert cosine law for $k=0.1$ and 0.2 . The maximum specular reflection appears at the receiving angle equal to the incident angle. The specular reflection is limited in a comparatively narrow range.

The quantitative evaluation of highly lustered fabrics can be made by dividing its reflection into specular and diffuse components (See Fig. 16). Moreover, because the distribution of the specular reflection is known such a receiving angle is obtainable as the specular reflection is as large as the diffuse reflection.

The specular component can easily be separated at this receiving angle. On the surface of the elliptical cylinder, $x$ corresponding to this angle is nearly equal to 1 . This means that a wide surface range of the elliptical cylinder is brilliant by the specular reflection. If the specular component is more brilliant, its luster seems much closer to that of metals. Consequently, the qualitative evaluation of the luster is lowered even though the quantitative evaluation is enhanced. Therefore, to evaluate a highly lustered surface, much attention

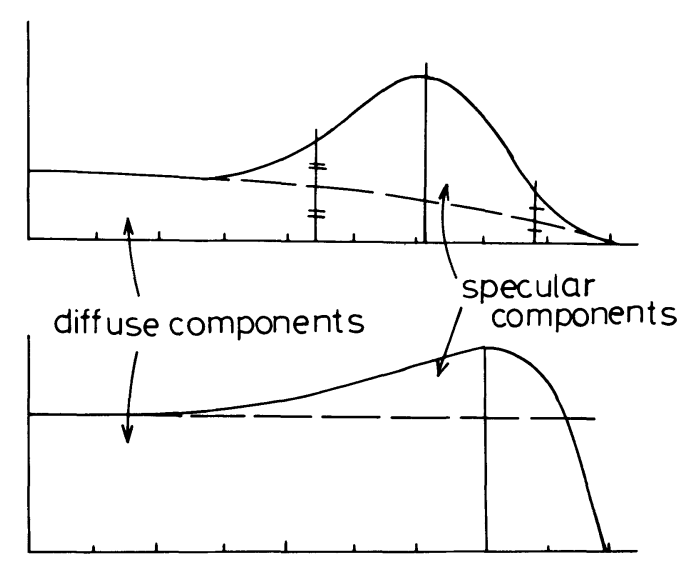

Fig. 16 Dividing specular components and diffuse components

Table 1 Details of Samples

\begin{tabular}{lllll}
\hline Samples & Weave & Yarn & $\begin{array}{l}\text { Count } \\
\text { (Picks } \times \text { ends/inch) }\end{array}$ & Color \\
\hline acetate satin & weft satin & bright $75 \mathrm{~d} \times 120 \mathrm{~d}$ & $272 \times 86$ & blue \\
rayon muslin & plain & semi dull 30/1 $\times 30 / 1$ & $89 \times 60$ & blue \\
glass fiber fabric & & DE $1501 / 01 \mathrm{Z} \times \mathrm{DE} 1501 / 4-80$ & $58 \times 32$ & brown \\
\hline
\end{tabular}


should be paid on two parameters, $k$ and $x$.

With increasing $k$ from 0.4 to 0.8 , the specular component decreases its maximum value and is distributed in a wider range of the receiving angle. The calculated value of the diffuse component is largely different from that of the Lambert cosine law, but little from the measured values. This may be because of insufficient assumptions of the optical diffusion in the stratum. Therefore, to evaluate the luster quantitatively, a procedure is proposed to draw a parallel line to the abscissa at the receiving angle of zero degree and divide two components. When $k$ is large, the surface of the elliptical cylinder which reflects the specular component is in a narrow range, and the luminance intensity is locally high and small surfaces of high luminance are widely distributed. As the surface area at which the luminance intensity of the specular reflection is larger than that of the diffuse reflection is undetermined for textile fabrics, no definite conclusion is deduced here. On the basis of the simplified model, the value of $x$ is calculable which corresponds to the receiving angle at which the specular reflection is more intense than the diffuse reflection. Therefore, such area can be calculated.

The quality of gloss is considered to be dependent on how the small surface is distributed, where the luminance intensity of the specular reflection is higher than that of diffuse reflec- tion. From our experimental results, $k$ of $0.2-0.3$ gives the preferable quality.

\section{Conclusion}

Approximate calculation of the specularly reflected light distribution curve is made by a simplified model having regular arrangement of elliptical cylinders. The results are much similar to the distribution curves obtained from textile fabrics, and are similar to measured results. So, it is confirmed that the simplified model is practically useful to analyse the gloss of textile fabrics.

\section{Literatures Cited}

[1] Nihira, Tsuboi, Gunji; J. Text. Mach. Soc. Japan, English edition, 26, T17, (1980).

[2] Gunji, Nihira, Tsuboi; J. Text. Mach. Soc. Japan, 24, T33 (1971).

[3] Tsuboi, Nihira, Gunji; J. Text. Mach. Soc. Japan, 25, T19 (1972).

[4] Tsuboi, Nihira, Gunji; J. Text. Mach. Soc. Japan, 27, T110 (1974).

[5] Nihira, Tsuboi, Gunji; J. Soc. Fiber Sci. Tech. Japan, 32, T23 (1976). 